Meta

Journal des tradlucteurs

Translators' Journal

\title{
Accès au métalinguistique naturel de la langue 2 par la traduction métalinguistique
}

\section{Claude Delmas}

Volume 33, numéro 3, septembre 1988

URI : https://id.erudit.org/iderudit/002533ar

DOI : https://doi.org/10.7202/002533ar

Aller au sommaire du numéro

Éditeur(s)

Les Presses de l'Université de Montréal

ISSN

0026-0452 (imprimé)

1492-1421 (numérique)

Découvrir la revue

Citer cet article

Delmas, C. (1988). Accès au métalinguistique naturel de la langue 2 par la

traduction métalinguistique. Meta, 33(3), 351-355.

https://doi.org/10.7202/002533ar d'utilisation que vous pouvez consulter en ligne. 


\section{ACCÈS AU MÉTALINGUISTIQUE NATUREL DE LA LANGUE 2 PAR LA TRADUCTION METALINGUISTIQUE}

Claude Delmas

Université de Paris III, Paris, France

Nous voudrions, ici, prendre le contre-pied de la position de G. Steiner (1975) et déclarer qu'en ce qui nous concerne en tous cas la traduction apporte une contribution non négligeable tant au plan théorique qu'au plan de la didactique. Ajoutons qu'il serait bien étonnant que la linguistique en retour n'apporte rien à la traduction.

Nous avons choisi un domaine de la grammaire que nous appellerons « le métalinguistique naturel ". En analysant ce pan de la grammaire, nous tenterons de montrer que la traduction fournit un instrument capital dans la mesure où elle peut prendre le relais du discours théorique et peut offrir une expression pédagogique à ladite théorie. Nous nous situerons dans le cadre théorique de la métaopération tel qu'il est présenté dans la Grammaire linguistique de l'anglais ou dans $T R E M A, \mathrm{n}^{\circ} 8,1983$.

\section{ACCÈS À LA MÉTALANGUE NATURELLE PAR LA MÉTHODE DES INCIDENTS}

Nous évoquerons la problématique des «incidents 》 pédagogiques de l'activité traduisante telle que la présente J. Guillemin-Flescher dans sa Syntaxe comparée du français et de l'anglais. L'existence même des incidents dans l'activité traduisante et surtout leur prise en compte sur le plan pédagogique constitue un enrichissement certain. Nous n'avons pas à enseigner les opérations mentales structurantes comme le signale A. Culioli dans Why Teach How to Learn to Teach What is Best Learnt Untaught (1979), mais il convient de conduire l'apprenant à résoudre le problème du "marquage " de l'opération dans le linéaire. Les "incidents » qui nous préoccupent proviennent de deux sources.

1. La non-reconnaissance de l'opération elle-même.

2. La non-explicitation de l'opération dans le linéaire.

Une stratégie traduisante peut faire affleurer les phénomènes enfouis ; on peut alors réinvestir les propriétés d'un incident :

(1) A : Y a-t-il de la vie sur d'autres planètes?

B : Sur les plus rapprochées de nous, probablement pas.

(2) B : *On THESE nearest to us, probably not.

L'incident apparaît en (2). Pour justifier THESE les étudiants qui ont choisi cette solution font appel à NEAREST TO US. Ces étudiants s'appuient sur l'opposition caricaturale (THIS : proximité, THAT : éloignement). L'étudiant croit faire une "faute " à cause de la grammaire. Rectifions tout de suite. L'incident est dû à une grammaire incomplète. Une grammaire qui par exemple ne s'occuperait que de mettre en relation des éléments lexicaux sans se préoccuper du statut de la relation. Ce genre d'incident conduit à réfléchir sur l'activité pédagogique traduisante. La traduction doit-elle se limiter à une activité corrective ? J.-R. Ladmiral critique un tel programme en parlant des dan- 
gers du « silence métalinguistique ». Suffira-t-il de dire à l'étudiant qu'il fallait THOSE? Il convient en fait d'avoir accès à cette "chimie " de la structuration où l'on ajoute, où l'on retranche ce qui vient spécifier le statut de la relation. En d'autres termes, il y a lieu de lutter contre le SQUELETTE LEXICAL de l'énoncé. Rappelons que l'étudiant est généralement obnubilé par le lexique, ce qui le conduit à ne pas rendre justice à la structuration. D'ailleurs la tradition l'encourage dans cette voie en parlant de " mots vides". Une véritable hyperlexicalisation devient la source de ses erreurs. Nous pourrions schématiser comme suit :

sur CELLES qui sont les plus PROCHES de nous

\section{$\varnothing$ CELLES $\varnothing \varnothing \quad \varnothing \varnothing$ PROCHES $\varnothing \varnothing$}

\section{THESE}

Par cette lexicalisation outrancière l'étudiant délaisse l'opération NEAREST TO USS. Sans entrer dans le détail on peut dire que THOSE a ici pour rôle de signaler la complexité de la structure née de l'opération NEAREST TO US. Une propriété ÊTRE PROCHE est détournée de sa fonction première à des fins de limitation. Nous ne sommes plus dans le domaine de la référence directe mais celui des opérations de comparaison, nous sommes au niveau des opérations, celui du métalinguistique naturel.

Une opération seconde, donc, impliquant une restriction sur une classe à des fins de spécification de validité. Autant d'opérations, autant de distanciation par rapport à l'origine de l'énoncé. THOSE marque ce poids énonciatif accru à partir de l'origine constitutive de l'énoncé. THOSE ne parle plus du réel. On songera à la définition de $\mathbf{R}$. Lafont, dans le Travail et la langue :

(...) observer la façon dont le système de la langue tout entier se décharge de la production du sens pour décrire son propre fonctionnement, ce qui est la fonction métalinguistique, ou métapraxémique.

On ne sera pas étonné d'apprendre que ce sont justement ces dits métalinguistiques qui posent problème $(B E+I N G, T H E$, etc.).

\section{LE CALQUE TEMPORAIRE PÉDAGOGIQUE : DIVERGENCES}

Après le squelette lexical, la tentation du calque peut tout à la fois constituer une catastrophe et une aubaine pour le pédagogue. Encore une fois si le métalinguistique naturel est éludé, le danger est patent :

(3) Allez, venez $/ *$ Go, come.

(4) Allons donc! /*Let's go then!

Il convient de voir que l'impossibilité du calque nous met en garde contre une conception trop "notionnelle» des éléments de la relation. Pour des exemples du type (4) G. Deléchelle, qui préfêre le terme de métatraduction, nous prévient qu'il convient de faire une analyse en profondeur. Ainsi le morphème -ONS constitue la trace d'opérations polémiques. La traduction de (4) fait affleurer cette opération polémique.

(5) Not a bit of it.

(6) Nonsense!

Avançons les énoncés suivants :

(7) Allez, je vous écoute.

(8) *Go, I'm listening.

(9) Go on, I'm listening to you. 
En français nous avons une trace de la relation pragmatique -EZ. Il s'agit d'une véritable injonction : "ALLEZ = avancez dans votre discours 》. Cette avancée dans le discours est matérialisée en anglais par $O N$. Parfois la trace pragmatique reste très abstraite et ténue :

(10) J'ai bien souffert allez!

(11) I've been through a lot, believe me.

(12) Why. You are not afraid, are you!

Gérard Deléchelle analyse cette structure comme une remise en cause, cette opération qui implique l'énonciateur aussi bien que le destinataire se retrouve dans le -ONS de VOYONS.

Là encore on ne peut coller au programme de sens strict. Il convient de restituer les opérations qui viennent moduler. Ce sont donc les relations qu'il convient de viser pédagogiquement ; une première conclusion s'impose dans l'ordre de la didactique :

1. Traduction sans métalinguistique naturel : incidents.

2. Métalinguistique sans traduction (abstraction trop élevée).

3. Traduction temporaire à visée métalinguistique (hypothèse raisonnable).

III. PARENTHĖSE MÉTALINGUISTIQUE ET CONTRASTIVE

On pourra penser que la proposition 3 ne correspond pas à une grande innovation. Dans la tradition certains auteurs ont tenté de donner consistance à cette proposition, en utilisant le calque :

(13) There are some beautiful houses in our street also.

(14) Hay (allí estan (+)) casas hermosas en nuestra calle tambien.

L'intention est ici louable mais l'auteur n'a pas vu ce simple fait que THERE et $B E$ ne gardent plus toutes leurs propriétés après mise en relation ; rien d'étonnant à cela après la lecture de Tesnière. La conséquence est que dans ce cas THERE ne peut renvoyer à $A L L I ́$. On retrouve le même problème avec :

(15) Here is a good racket for you.

(16) He aquí (aquí está) una raqueta para $V d$.

Ceci montre les précautions qu'il convient de prendre lors de l'utilisation d'un calque temporaire et agrammatical. L'agrammaticalité du calque doit orienter l'étudiant vers une solution et non pas donner à penser que ce calque dispense d'un calcul correcteur. Voici un autre exemple :

(17) Ya tengo el permiso de conducir.

(18) J'ai (déjà) le permis de conduire.

(19) A : ¿Llevaron tambien a los niños?

B : Sí, porque ya no tenían colegio.

(20) A : Ont-ils emmené les enfants aussi ?

B : Oui, parce qu'ils (déjà) n'avaient plus d'école.

(21) Ya ves, terminando de instalarnos, ¿te gusta el piso ?

(22) (Déjà) tu vois, en train de finir de nous installer. L'appartement te plaît ?

Nous avons un calque avec DÉJÀ mis entre parenthèses. On donne à penser que l'espagnol utilise un DÉJÂ redondant explétif. Il s'agit, on l'aura compris, d'un DÉJÀ métalinguistique. L'espagnol signale que le travail de structuration cohésive effectué par l'énonciateur est chose faite, acquise. On comparera avec :

(23) $Y A$ voy

(24) I'm coming

L'action dans l'extralinguistique n'est pas faite, mais la thématisation de la relation prédicative, elle, est DÉJẦ assurée. C'est le travail de mise en relation qui est déjà fait. Il s'agit de structuration acquise, donc de métalinguistique. 


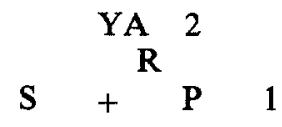

Dans le fond YA est la dernière opération effectuée par l'énonciateur, c'est donc la dernière opération qui compte, c'est elle qui domine, en conséquence, c'est plutôt la relation décalée $\mathbf{S}+\mathbf{P}$ qu'il aurait fallu mettre entre parenthèses.

$$
\underset{2}{\text { Énonciateur }} \mathrm{YA}_{1}(\mathrm{~S}+\mathrm{P})
$$

Ceci montre encore une fois que la traduction calque reste insuffisante si le relais métalinguistique naturel n'est pas atteint.

\section{CALQUE TEMPORAIRE ET CONVERGENCES}

La proposition 3 de notre récapitulation nous invite également à utiliser la traduction comme stratégie d'accès lorsque les convergences sont possibles, même si dans une étape subséquente il convient d'abandonner cette traduction temporaire pour des raisons stylistiques. On pourra donc recourir à cette stratégie dans la mesure où parfois les métaopérations fonctionnent de manière relativement homologues.

Mais :

(25) *Où est une chandelle?

(26) *Where's a candle? (dans le sens de se trouver)

(27) Où est la chandelle ?

(28) Where's the candle?

Il est aisé de voir pourquoi l'opération WHERE est antinomique avec l'opération $A C A N D L E$. Il y a là moyen de sensibiliser les étudiants avec le métalinguistique. $T H E$ est un commentaire sur l'état de la structure nominale. Une fois sensibilisés on peut demander à ces étudiants de traduire le métalinguistique ailleurs dans d'autres types de structures. Voici une expérience qui a été faite avec des étudiants de première année, à partir d'un exemple dû à $\mathrm{H}$. Adamczewski :

(29) You have been drinking whisky! (entame)

Solutions métalinguistiques proposées :

(29a) $\mathrm{Tu}$ as bu du whisky!

(29b) Toi, tu as bu du whisky!

(29c) Ma parole, tu as bu du whisky!

(29d) Toi, mon vieux, tu as bu du whisky!

(29e) Dis donc, tu as bu du whisky!

(29f) À ce que je vois, tu as bu du whisky!

(29g) Pas de doute, tu as bu du whisky!

(29h) Tu as encore bu du whisky!

Pour le détail de l'analyse nous renvoyons aux travaux de H. Adamczewski. Soulignons que la reprise marquée en anglais par $B E+I N G$ apparaît nettement dans certaines traductions TOI TU, DONC, ENCORE. Soulignons également la nette prise de position marquée par MA PAROLE, PAS DE DOUTE, Ä CE QUE JE VOIS.

Dans ce cas c'est le statut de relation dominée que les étudiants ont cherché à rendre. Rappelons qu'une convergence continuée dans l'agrammatical peut rendre des services.

(30) *John's being ill is true.

(31) * Ma conviction que Jean est malade est vraie.

Après les Kiparski mettons l'accent sur le fait qu'une conviction peut être bien ou mal fondée mais elle ne peut être vraie. Nous nous situons alors dans une négation méta- 
linguistique, puisque la proposition ne peut être validée par l'état du monde (le vrai et le faux) mais uniquement par la structuration factive métalinguistique bloquée par l'opérateur CONVICTION.

\section{ACCÈS À LA NÉGATION MÉTALINGUISTIQUE}

Lorsque la structuration opère un blocage formel et sémantique l'autonomie référentielle subit une limitation qui peut aller jusqu'à la négation. Cette négation métalinguistique permet par la distanciation qu'elle implique des variantes métalinguistiques dans la traduction :

(32) I'm not going with you!

(33) Non, non, je ne vais pas avec toi !

(34) Pas question que j'aille avec toi!

(35) Je refuse $d$ 'aller avec toi!

Quand la négation y est plus sensible, une distanciation plus grande est permise :

(36) I've been painting the garage!

(37) J'ai peint le garage !

(38) J'ai les mains sales !

L'énoncé (37) est une traduction faiblement métalinguistique de (36). L'énoncé (38) par contre est une traduction fortement métalinguistique.

(39) She was dressed as a traffic warden.

(40) She was dressed like a traffic warden.

Glosons M. Swan : $A S$ renvoie à de la conformité au réel ; $L I K E$ au contraire implique une négation sous-jacente "she is wearing a peculiar outfit ». Les traductions mettront ceci en évidence :

(41) Elle était habillée comme un clown.

(42) Elle était habillée comme l'as de pique.

(43) Elle est mal habillée.

(44) Elle n'a aucun goût pour s'habiller.

(45) Elle ne sait pas s'habiller.

(46) Elle s'habille n'importe comment.

(47) Elle s'habille à la six-quatre-deux.

(48) Elle s'habille comme c'est pas possible.

(49) Elle a l'air déguisée.

(50) Elle est habillée à la décrochez-moi-ça.

La négation métalinguistique permet toute une famille métalinguistique ; la multiplicité prend le relais de la négation métalinguistique invariante. On comprendra que l'énoncé (39) avec $A S$ lié au conforme épouse l'extralinguistique et ne permet que le seul calque du réel :

(51) Elle était habillée en contractuelle.

Les traductions viennent renforcer les prédictions de l'analyste. Si les traductions foisonnent dans le métalinguistique naturel c'est bien que le thématique a rompu les liens (ou les a en tous cas distendus) avec la conformité au réel. Le concept de PHASE 2 permet tout un champ pédagogique qui réconcilie théorie et pratique.

La structuration métalinguistique naturelle qui reste si souvent délicate à faire percevoir par nos étudiants se trouve largement représentée et explicitée dans le domaine de la traduction. Parmi toutes les traductions possibles certaines sont plus métalinguistiques que d'autres. L'intérêt de la traduction métalinguistique réside dans le fait que loin d'enfermer l'apprenant dans un modèle qu'on lui assène, elle lui permet de redécouvrir pour son compte une opposition qui traverse toute la grammaire ; accordant 
plus d'autonomie à l'apprenant on peut gager que son apprentissage en sera moins artificiel et certainement plus motivé.

\section{BIBLIOGRAPHIE}

ADAMCZEWSKI, H. (1982) : Grammaire linguistique de l'anglais, Paris, A. Colin.

ADAMCZEWSKI, H. (1983) : "Analyse métaopérationnelle de l'anglais", TREMA, no 8.

ADAMCZEWSKI, H. (1975) : "Le montage d'une grammaire seconde ", Langages, no 39

FALLARD, M. (1980) : la Traduction de l'anglais, Lille, Presses universitaires de Lille.

CULIOLI, A. (1979) : "Why Teach How to Learn to Teach what is Best Learnt Untaught ", Cahiers Charles $\boldsymbol{V}$.

DANON-BOILEAU, L. (à paraître) : "That Is the Question ", Modèles linguistiques.

DELÉCHELLE, G. (1983) : "Remarques sur la contrastivité », CIEREC, no 39.

DELMAS, C. (à paraître) : "Négation métalinguistique comme parenthèse invisible ", Contrastes.

GUILLEMIN-FLESCHER, J. (1981) : Syntaxe comparée du français et de l'anglais, problèmes de traduction, Ophrys.

LADMIRAL, J.-R. (1979) : Traduire : théorèmes pour la traduction, Paris, Payot.

LAFONT, R. (1978) : le Travail et la langue, Paris, Flammarion.

L.AKOFF, G. and M. JOHNSON (1980) : Metaphors we live by, Chicago.

Z'EMB, J.M. (1975) : "Réflexions éristiques et heuristiques sur le 'tertium comparationis' ", Langages, n’ 39. 